\title{
Manifesto for Digital Social Touch in Crisis
}

\author{
Carey Jewitt ${ }^{1 *}$, Sara Price ${ }^{1 \dagger}$, Jürgen Steimle ${ }^{2 \dagger}$, Gijs Huisman ${ }^{3 \dagger}$, Lili Golmohammadi ${ }^{1}$, \\ Narges Pourjafarian ${ }^{2}$, William Frier ${ }^{4}$, Thomas Howard ${ }^{5}$, Sima Ipakchian Askari ${ }^{6}$, \\ Michela Ornati ${ }^{7}$, Sabrina Panëels ${ }^{8}$ and Judith Weda ${ }^{9}$ \\ ${ }^{1}$ UCL Knowledge Lab, Department of Culture, Communication and Media, IOE, University College London, London, \\ United Kingdom, ${ }^{2}$ Augmentation Human Lab, Department of Computer Science, Institute X, Saarland University, Saarbrücken, \\ Germany, ${ }^{3}$ Perceptual Intelligence Lab, Human-Centered Design Group, Delft University of Technology, Delft, Netherlands, \\ ${ }^{4}$ Ultraleap, Bristol, United Kingdom, ${ }^{5}$ Rainbow Lab, INRSA, University of Rennes, Rennes, France, ${ }^{6}$ Human-Technology \\ Interaction Group, Eindhoven University of Technology, Eindhoven, Netherlands, ${ }^{7}$ Institute of Digital Technologies for \\ Communication, Faculty of Culture, Communication and Society, Università della Svizzera italiana, Lugano, Switzerland, ${ }^{8}$ Sensory \\ and Ambient Interfaces Lab, Institute CEA LIST, University Paris-Saclay, Paris, France, ${ }^{9}$ Human Media Interaction Group, The \\ Digital Society Institute, University of Twente, Twente, Netherlands
}

This qualitative exploratory research paper presents a Manifesto for Digital Social Touch in Crisis - a provocative call to action to designers, developers and researchers to rethink and reimagine social touch through a deeper engagement with the social and sensory aspects of touch. This call is motivated by concerns that social touch is in a crisis signaled by a decline in social touch over the past 2 decades, the problematics of inappropriate social touch, and the well documented impact of a lack of social touch on communication, relationships, and well-being and health. These concerns shape how social touch enters the digital realm and raise questions for how and when the complex space of social touch is mediated by technologies, as well the societal implications. The paper situates the manifesto in the key challenges facing haptic designers and developers identified through a series of interdisciplinary collaborative workshops with participants from computer science, design, engineering, $\mathrm{HCl}$ and social science from both within industry and academia, and the research literature on haptics. The features and purpose of the manifesto form are described, along with our rationale for its use, and the method of the manifesto development. The starting points, opportunities and challenges, dominant themes and tensions that shaped the manifesto statements are then elaborated on. The paper shows the potential of the manifesto form to bridge between $\mathrm{HCl}$, computer science and engineers, and social scientists on the topic of social touch.

Keywords: touch, social touch, digital touch, sensory, haptics, design, manifesto, interdisciplinary research

\section{INTRODUCTION}

This qualitative exploratory research paper presents a Manifesto for Digital Social Touch in Crisis (Figure 1). The manifesto's call - to rethink and reimagine digital social touch through a deeper engagement with the social and sensory aspects of social touch, is motivated by concerns that social touch is in crisis (both historically and looking forward). There has been a significant decline in social touch over the past 2 decades with an increase in a culture of "low-no-touching" (Linden, 2015), a decrease amplified by Covid-19 (Field et al., 2020). The problematics of inappropriate social touch, abusive social touch, and the ethics of social touch are well documented (e.g., Field, 2014). This raises 
MANIFESTO FOR

$\begin{array}{lllllll}D & \text { I } & \mathbf{G} & \mathbf{I} & \mathbf{T} & \mathbf{A} & \mathbf{L}\end{array}$

$S O C$ I A L

$\mathrm{T} \mathrm{O} \mathrm{U} \mathrm{C} \mathrm{H}$

IN CRISIS

This manifesto is for designers and developers working on digital touch across academia and industry. It is a vision designed to challenge and provoke debate, raise awareness, incite questions, inspire and direc research and design on the social and sensory aspects of the digital design of touch

Human touch is at risk. Of disappearing. Neglected touch. Low touch. No touch. Not enough touch. Touch hunger. Skin starvation. Touch deprivation. Remote. Distant. Missing. ///Flip -Switch/// Too much touch. Intrusive. Unwelcome. The wrong kind of touch. Fee the cacophony, the weight of fears for technological touch - but don't forget the good dreams.

\section{MAKE SOCIAL TOUCH CENTRAL}

Human touch matters! Hone in on social touch. Include 'touch matters' at the heart of digital communication. Amidst conflicting social concerns and heart of digital communication. Amidst conficting social concerns and and promises for social touch. Give life back to the digital: Feel the pulse.

\section{DESIGN TOUCH FIRST, TECHNOLOGY} SECOND

Touch is at risk in technology. Technology (alone) is not the solution. We need technologies to better realize digital touch. Goals for digital touch should be set by something more than technological availability. Amplify interdisciplinarity to PRESS RESET. Rebalance the dialogue between the social, sensory, tactile aesthetic and technological drivers underpinnin digital touch development. Bring nuance to a collective imaginary of future touch.

\section{DEMOCRATISE TOUCH: DON'T LOCK IT IN}

Touch is political. Touch is infused with power. We are positioned to touch through socio-economic and socio-cultural drivers. Let's interrogate how digital design reshapes touch needs and norms. Digital touch needs to be felt conversation. Everyone is qualified to have a say on what digital touch might be/become. The touch etiquettes of the 20th century will not suffice OPEN up 'opportunity spaces' to AVOID imaginations of touch getting 'locked in'. DESIRE HETEROGENEITY. Just say no to 'homogenised touching'. Amplify diversity through haptic encodings.

\section{PROTECT TOUCH: KEEP TOUCH PRIVATE} AND SECURE BY DESIGN

Touch is intimate. Touch reveals myself and my boundaries. When I touch what do I convey about myself? What did I feel of you? Am I identify-able? Did you feel it was me? Can I touch back? Touch overload. Touch Space Invader Unauthorised touch. WARNING. Alert! Deceptive touch. Hacked feel. Fak Touch 'Retouchée'. Guarantee me control of my digital touch patterns and preferences. Record. Share. Replay. Mix. Consent. I own my digital touch. Don't wait to tame the haptic 'Wild West'. Develop a haptic business model beyond haptic-monitoring. Let the user decide when, how and where technology touches.

5. MOVE BEYOND VIBRATION: FEEL BEYOND THE HABITUATED

Pay closer attention to the FEEL of touch. Re-encode touch sensations. Create landmarks of felt resistance in the tactile terrain. Materialise touch! ROUGH it up - WARM it up. Give us texture. Soft, bouncy, warm and comfortable How about a bit of give and take-Material reciprocity beyond the limits of the slick or smooth. Give me a digital touch I don't expect. Negotiate new tactile rhythms. Make time for touch. Ambient S-L-O-W touch. Enrich the shallow vibrating utilitarian feel venture beyond the hand, beyond the skin shallow digital touch thrive.

\section{FOSTER EXPLORATION OF MEANINGFUI} TOUCH EXPERIENCES

PERSONALISE TOUCH. Design for a plurality of touch preferences and sensitivity thresholds. Keep it indoors, get mobile, take digital touch outdoors? DESIGN a varied touchy terrain. Include more options. LEAVE SPACE for MUTUAL touch creation. STRETCH the embodied limits of touch socially and functionally to explore new possibilities. A customisable tactile landscape to support the development of social touch languages Give the user felt feedback to sense their own touch and handle when and where touch lands. Give digital touch value: calculate its biological cost. Allow social touch to extend beyond the immediately comfortable everyday zone.

\section{REMAKE, DON'T ONLY REPLICATE!}

Free digital touch from the limits of its analogue reflection and let go. Confound and RECALIBRATE touch. Challenge the status-quo. Foster alternative visions. Engage with unfamiliar touch, prepare to touch newly. Trash the touch-screen devoid of feedback. Translate the rich language of non-human touch into the tactile landscape. Rename touch. Reconfigure reliance on visuality. Take a leaf from nature, mimic reality as a stepping stone to remake touch. Or JUMP into the water and wade to a new bank to stone to remale
refresh touch.

\section{MANAGE GREAT TECH-XPECTATIONS}

Frame users' techy touch expectations. Communicate and contextualise digital touch. Avoid the feel of disappointment. Digital touch is a long-term endeavour. Temper commercial HYPE. No need to over-promise - 'LIKE NOTHING EVER FELT BEFORE!' Keep possibility alive within an honest sense of promise. Metaphors and imagination can bridge a user to unexpected social, sensory and digital touch. Managing expectations is an important step social, sensory and
towards adoption.

\section{DEVELOP OPEN TOUCHY TOOLS}

We need diverse collaborative tools, libraries and archives, technical tools, thinking tools and sensitizing tools to expand touch in the digital realm. Shared tools that can travel between users, designers, developers, researchers. Make. Try. Explore. Tools to inspire and educate and bring potential users mito the digital touch dialogue. Generate new touch metaphors. Grab at it, pinch and mould it, make our vocabularies work so we can hone new relationships between language and technology to create touch sensations, talk me into feeling it. COMMUNCATE! We need to understand each other better.

\section{KEEP SPECULATING}

Revisit touch pasts. Interrogate touch presents. Take an E-X-P-A-N-D-E-D view of touch. Forecast the influence of digitally-mediated touch on social interaction. Explore the texture in the space between touchy Dystopias

FIGURE 1 | A Manifesto for digital social touch in crisis.

questions of the agency, control, and regulation of social touch (in workplaces, schools, healthcare settings etc.), much of which are entangled with the politics of power and gender (e.g., \#MeToo) in both institutional and domestic settings (Field, 2002; Halley, 2007; Owen and Gillentine, 2011; Green, 2017; Pihkala et al., 2019). Despite this social touch remains central to human experience (Bull et al., 2006; Field, 2014), communication
(Gallace and Spence, 2010), and relational, psychological and physiological well-being (Jakubiak, and Feeney, 2017). The impact of a lack of social touch on communication, relationships and well-being and health is well documented as having demonstrable negative connotations (Gallace and Spence, 2010; Field et al., 2020). The specific and immediate consequences of the Covid-19 pandemic for social touch have amplified 


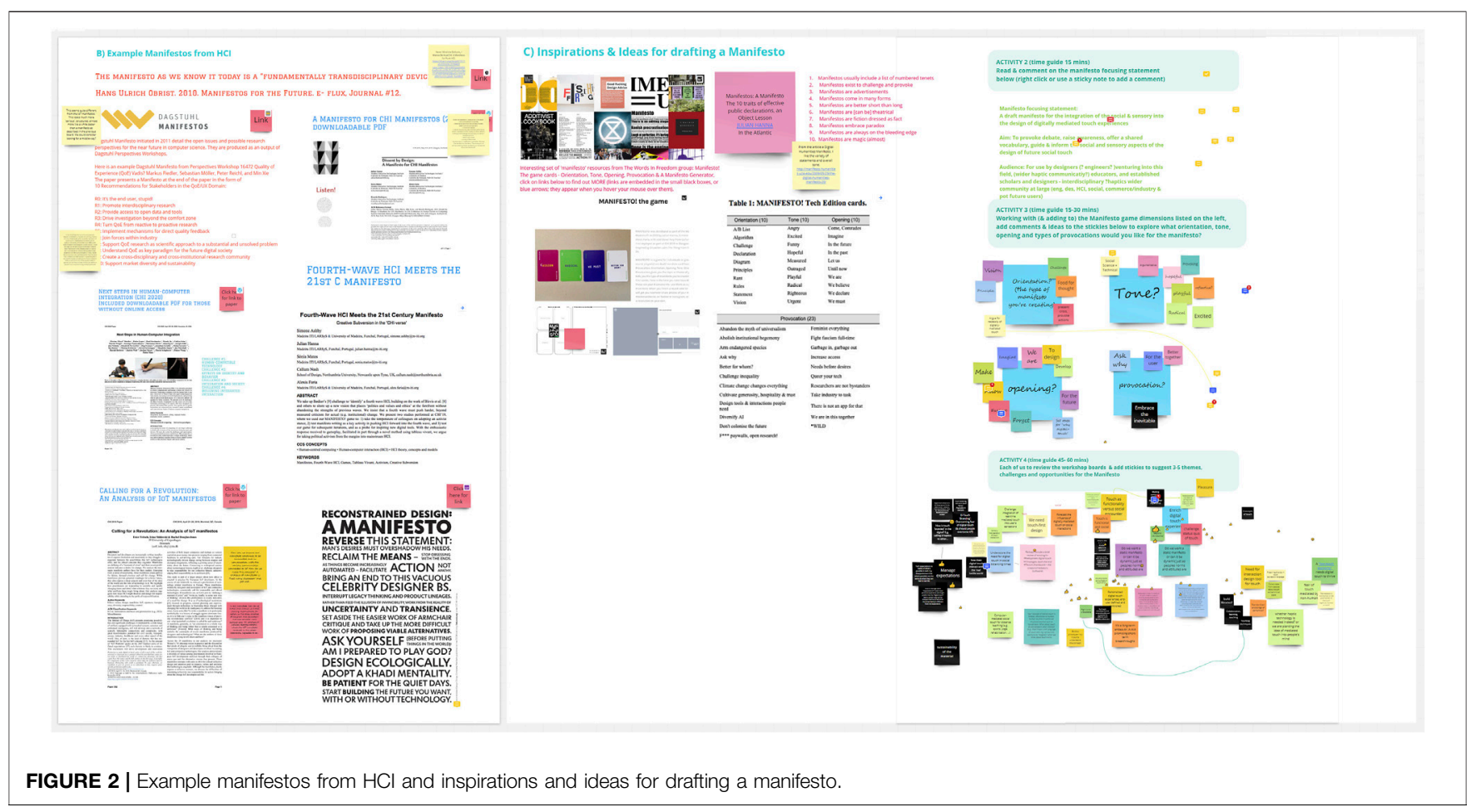

assertions in the media, governments, and health care organizations that social touch is in crisis. In addition, restrictions and regulations prompted by Covid-19 have unsettled and shifted social touch etiquettes, social norms and expectations of social touch (Green and Moran, 2021).

These concerns bleed into and shape the digital realm and raise questions of how and when the complex space of social touch is mediated by technologies and the societal and sensorial implications of doing so. The manifesto was initiated in autumn 2020 during the flux of the Covid-19 pandemic, a point of emergence from (and shortly a return to) global lockdown. A global event that has perhaps more than any other recent one, highlighted the human need for social touch and the paucity of visual technologies for communication in response to this need has felt palpable: unleashing hopes and fears for both social touch and its digital futures. Social touch is a complex space for technology to mediate. Social touch is increasingly central to the digital futures imagined within Human-computer interaction (HCI) (Hoggan, 2013; Huisman, 2017). We are at a point where despite the success of haptics as a field, and its potentials for social touch. Questions about the successful replication of social touch in mediated environments remain (Haans et al., 2014; Willemse et al., 2017; Askari et al., 2020; Ipakchian Askari et al., 2020) including how technology should be deployed and used for social touch. The manifesto thus speaks back to the technological landscape and asks, is digitally mediated social touch itself in a moment of crisis?

The Manifesto for Digital Social Touch in Crisis is an outcome of a series of interdisciplinary collaborative workshops, initiated at Eurohaptics 2020, on the social and sensory aspects and challenges of designing digitally mediated social touch. The workshops took place over 6 months with participant collaborators from computer science, design, engineering, HCI and social science within industry and academia. Drawing on the research literature and the expertise of these collaborators, the manifesto takes a broad view of design and development. In doing so it offers routes to navigate the technological realities and promises for social touch amidst conflicting social concerns (e.g., the loss of cultural variations in touch practices through the homogenized effect of hegemonic technology powers or standardization, the social effect of socio-economic divides in access to digital social touch, the new social norms that may emerge through digital social touch) and uncertain futures (e.g., how digital social touch devices will enter the global market, models for storing and sharing digital social touch related data, and what future touch sensations maybe realized digitally and the possibilities for the customization of digital social touch).

The manifesto form has traditionally been taken up and used in politics, the arts (e.g., The Manifesto of Tactilism by the Futurist artist Marinetti, 1921) and design (e.g., Design Justice Network Principles, 2018). The manifesto is an alternative genre and comes in many forms, usually in response to a crisis, and is an urgent call for change articulated through an eye-catching list of challenging and provocative statements. Manifestos are typically short and sharp, often purposefully poetic and ambiguous, with a tone ranging from playful, excited or hopeful through to angry. Manifestos remain less common in $\mathrm{HCI}$ in comparison to other disciplines (Hanna et al., 2019), although their take up within HCI appears to have increased recently with the development of manifestos on various aspects of design (e.g., Design Justice Network Principles, 2018), specific technologies, such as Blockchain technology (Elsden et al., 2019) and the Internet of 
Things (reviewed in Fritsch et al. (2018)), and futures more generally (e.g., the Dagstuhl Manifesto series initiated in 2011). The manifesto as we know it today is also a "fundamentally transdisciplinary device" (Obrist, 2010).

While manifestos may emerge from or be informed by research, they are not a scientific outcome. Manifestos aim to provoke. The purpose of manifestos is "prompting new ideas by temporarily liberating scholars from the confines of careful speech and rational argument" - to offer a chance to question and imagine possible futures (Hanna et al., 2019:2), and this, it is argued, has the potential to "give new life to" HCI (ibid). They usually emerge from uncertainty, set out to define a moment of crisis and "invite us, loudly, to some new way of thinking” (Caws, 2001: xxix): in short, they signal a desire for change. The Manifesto for Digital Social Touch in Crisis offers a set of 10 statements characteristic of the manifesto form, using a playful variety of poetic, ambiguous and provocative formulations to challenge the reader. Phrases such as "///Flip -Switch///" for example, mark the binary extremes that dominant the response to digital social touch technologies as either "lacking/absent" or "overwhelming/wrong"; "Feel the cacophony" is used to point to the noisy debates of what digital social touch should be, as well as the non-stop sound of vibrating devices that we live with; and “Tech-Xpectations," is a play on Charles Dicken's novel Great Expectations, which is ultimately about the eventual triumph of good over evil. It takes up the challenge to articulate the "struggle to negotiate between the possibilities that technologies offer, and the concerns that they engender" (Fritsch et al., 2018: 1) and proposes paths toward future roadmaps or directions for digital social touch in society.

The qualitative work (including the manifesto) presented in this article is explorative rather than experimental. Qualitative research is defined as an iterative process through which improved understanding of the scientific community is achieved by making new significant distinctions resulting from getting closer to the phenomenon studied (Aspers and Corte, 2019). Here, the purpose of iteration is as a reflexive process to spark insight and develop meaning (Srivastava and Hopwood, 2009). Using this approach, we sought to understand the concepts, opinions, and experiences raised by the complex "real-world" challenges and opportunities faced by designers in relation to the social and sensorial aspects of designing digital social touch. Our focus, in line with the characteristics of qualitative research, was on building categories, patterns and themes from the ground up (inductive) to capture the participant collaborators' meanings - here in the innovative form of a manifesto. The manifesto is thus developed through and rooted in the interdisciplinary expertise of social touch designers/developers/researchers: a research outcome of a qualitative iterative design process (Sale and Thielke, 2018). Rather than providing a starting point for the manifesto, the literature on the contemporary landscape of social touch and haptics (reviewed below) situates the manifesto within the wider debates and challenges of haptics and serves to contextualise the concerns crystalized within the manifesto. The article sets out the process that shaped the manifesto development, the curation of its statements, and discusses the opportunities and challenges and the dominant themes and tensions that informed the manifesto.

\section{BACKGROUND}

The manifesto emerges in response to the contemporary landscape of social touch. It situates itself within the larger field of haptics, addressing both the study of human touch and technology that stimulates the senses of touch and motion (Hannaford and Okamura, 2016; Jones, 2018). Social touch refers to the many forms of touch in social communication - e.g., greetings, intimate communication, corrections (van Erp and Toet, 2015). It can comprise one or multiple sub-modalities: touch, temperature, itch, pain, and affective touch. We use the term 'digital touch' to denote digitally mediated touch sensations, digital social touch and haptics (both referring to the study of the human sense of touch, and its submodalities, touch, temperature, itch, pain, and affective touch, as well as the use of technology that stimulates the senses of touch and motion (Hannaford \& Okamura, 2016; Jones, 2018)) more broadly. We outline this landscape below.

\section{Social Aspects of Touch and Haptics}

Social touch is part of the human socio-affective, communicative repertoire in the form of interpersonal touch (Gallace and Spence, 2010; McGlone et al., 2014; Jewitt et al., 2020). Social touch, in the form of, for example, hugging, hand holding or stroking, plays a critical role in human development (Cascio et al., 2019), is related to improved overall well-being (Field, 2019), can reduce stress (Ditzen et al., 2007), blood pressure and resting heart rate (Light et al., 2005), and pain (Goldstein et al., 2016), plays a role in communicating emotions (Hertenstein et al., 2006), can enhance a positive mood (Debrot et al., 2013), and has positive effects on pro-social behavior (the Midas touch effect; Haans et al., 2014). Social touch is typically experienced as signaling intimacy and occurs particularly frequently between people in a romantic relationship (Guerrero and Andersen, 1991), or between parents and children (Chopik et al., 2014). Social touch is considered a cross-cultural phenomenon that, although different in form and in its embedding in social practices (see Carra et al. (2014)), occurs across the globe (Sorokowska et al., 2021). Variability in social touch practices can relate to differing environmental aspects (e.g., climate), cultural aspects (e.g., prevalence of religion), and social norms (Sorokowska et al., 2021). Moreover, individuals' history of touch interactions from early on in life influence how they experience touch in later life (Bales et al., 2018), and there are differences between individuals in how comfortable they are with touch in general, which can be captured by touch avoidance (Ozolins and Sandberg, 2009) or longing for touch questionnaires (Beßler et al., 2020). The Covid19 pandemic has made palpable that a lack of social touch can have negative consequences for well-being, with research into "touch hunger" (Field et al., 2020; Durkin et al., 2021) finding that the majority of people profoundly miss being touched by others. Prior research has highlighted the negative effects of prolonged absence of social touch on well-being (Field, 2010), and these findings are supported by research on other mammals (Ardiel and Rankin, 2010).

While the mechanisms behind the effects of social touch (also known as socio-affective touch) are not fully understood, they are 
assumed to involve an interplay between social, cultural, neural, and opioid factors (Cascio et al., 2019). For example, part of this interplay of factors involves the importance of social touch in the early life of infants to maintain homeostasis and bond with a caregiver, which through a process of reinforcement-learning cements touch as an especially intimate and salient social signal in later life (Fotopoulou and Tsakiris, 2017; Cascio et al., 2019). It is also suggested that there is a prominent role for C-Tactile afferents (CT-afferents) in social touch (Olausson et al., 2010; McGlone et al., 2014). These are low-threshold nerve-fibers that are particularly sensitive to slow stroking touches, that is the types of touch that are judged as particularly pleasant (Löken et al., 2009; Olausson et al., 2010) and considered to signal socially relevant touches. This further distinguishes "CT-touch" from touch involved in tasks such as object manipulation (Cascio et al., 2019). Effects of social touch, such as those on stress reduction, might thus be strongest when CT-optimal touch is applied (Morrison, 2016). Finally, there is also research to implicate opioids such as oxytocin (which are released during social touch) as accounting for positive effects of social touch such as pain reduction (Walker et al., 2017).

Work on the effects that social touch has on well-being and the role it plays in communication has inspired investigations into the use of haptic technology for social touch interactions (Huisman and Darriba Frederiks, 2013). The current state-ofthe-art in haptic technology showcases the efforts of designers and engineers to create compelling digital touch sensations by making creative use of existing or the design of new actuator technologies. In one strand of research, haptic technology is used to mediate human-human social touch with the aim to reproduce some of the effects of actual social touch (Haans and IJsselsteijn, 2006). For example, Haans and IJsselsteijn (2009) used vibrotactile feedback during an online chat conversation as a form of mediated digital social touch to investigate whether receiving this type of feedback would affect helping behavior by the recipient of the feedback (i.e., the Midas Touch effect (Crusco and Wetzel, 1984)). Although the authors found an initial effect of digital social touch on helping behavior similar to that of the unmediated Midas touch effect, later research indicated that this might reflect a potential confederate bias, where the effect only occurred when the confederate was aware of the experimental condition (Haans and Ijsselsteijn, 2014). Other studies have looked into how mediated social touch affects interpersonal trust (Erk et al., 2015), can be used to communicate emotions (Huisman and Darriba Frederiks, 2013; Rantala et al., 2013), and examined how textual tone affects the evaluation of mediated social touch (Ipakchian Askari et al., 2020). Embedding digital touch into social sensory contexts of established social touch communication remains complex and raises key design issues for effective mediated social touch.

Research closely related to work on mediated social touch looks at how touch could also serve as a modality for virtual agents and social robots to communicate with humans (Huisman and Darriba Frederiks, 2013; van Erp and Toet, 2015). Here, sensors are used to grant virtual agents or robots the ability to detect touch and haptic technology serves as a way for these artificial social agents to apply touch to humans. For example, Huisman et al. (2014) used vibrotactile actuators to enable a virtual agent in augmented reality to apply touches to a participant's upper arm, which was found to affect participants' perception of the agent's personality. In other research, where a social robot was used to apply touches to participants' upper arms, it was found that touches by the robot attenuated physiological stress responses induced by watching movie clips (Willemse and Van Erp, 2019). Similar work with robots and virtual agents has focused on the expression of empathy through robotic touch (Bickmore et al., 2010), the communication of emotions (Cang et al., 2015; Obrist et al., 2015), and on improving well-being (Block et al., 2021). The integration of digital social touch interactions in these kinds of artificial social agent systems is not straightforward, however, since multimodal cues, including, facial expressions and speech prosody, can overshadow the effects of touch (Bickmore et al., 2010). These efforts all fit within a broader view on affective computing where touch is seen as another modality that could be used to gather emotionally relevant information from users as well as to apply emotionally salient stimuli to users (Eid and Al Osman, 2015).

Efforts to mediate social touch through haptic technology or to enable artificial social agents to engage in social touch often have the aim of reproducing aspects of naturalistic social touch, the idea being that technologically mediated social touch can have positive effects on well-being (Huisman, 2017). With the Covid19 pandemic resulting in increased experiences of touch hunger (Field et al., 2020; Durkin et al., 2021), it has been suggested that haptic technology can help to alleviate touch hunger to some extent (Prattichizzo, 2021), for example, by enabling affective touch interactions over distance (den Dekker et al., 2021). How touch is conceptualised matters in shaping technical advancements, bringing opportunities and challenges for development and design and raising questions for how touch experience is reconfigured. Recent work on touch in VR, for example, suggests that while the area is dominated by notions of touch as replication and illusion, interpretive designs of touch can disrupt established "real world" socialities of touch and their renegotiation by users in the space of digitally mediated touch in VR (Price et al., 2021). However, current research into the exact effects of digital touch technologies on the reduction of Covid-19 induced touch hunger are non-existent.

\section{Touch Technology}

Haptic experiences and devices (using force, tactile, vibrotactile feedback) have been increasingly applied in HCI and the development of immersive touch experiences is now relatively commonplace. A recent survey (Saleme et al., 2019) details the range of commercially available haptic devices including wearables (e.g., Tesla Suit, Dexmo, ARAIG, KOR-FX and Subpac $^{1}$ ); handheld devices (e.g., Vibrotactile mice and

\footnotetext{
${ }^{1}$ Tesla suit available at https://teslasuit.ioDexmo available at https://www. dextarobotics.com/en-usARAIG available at https://araig.comKOR-FX available at http://www.korfx.comSubpac 101 available at https://subpac.com/subpac-101/
} 
joysticks), desktop devices (e.g., Phantom Omni (Danieau et al., 2012), and Ultrahaptics, (Limerick et al., 2019; Rakkolainen, et al., 2021)), and haptic chairs. Haptic technology has found its way into various commercial applications, such as gaming (Orozco et al., 2012; see also Parisi, 2018), virtual reality (Srinivasan and Basdogan, 1997), tele-operation (Hirche and Buss, 2012), haptic gears (Shah et al., 2014; Prasad et al., 2014), automotive interfaces (Harrington et al., 2018; Breitschaft et al., 2019), digital signage (Limerick et al., 2019), mobile (Kwon et al., 2010) and wearable devices (Pacchierotti et al., 2017; Parisi and Farman, 2019), augmented reality (Romanus et al., 2019) and (inevitably) sex toys (Döring and Pöschl, 2018; Liberati, 2017).

The industry standard for high-resolution touch input is capacitive touch contact sensing. Such sensors have been available inside rigid touch screens and mobile devices for a while. Technologies for haptic output can be subdivided into approaches for kinesthetic and for cutaneous feedback, the former delivering forces and torques and the latter delivering tactile skin sensations directly on the skin (e.g., vibration, skin stretch, thermal cues). Early haptic devices were mostly grounded and often focused on kinesthetic feedback (Culbertson et al., 2018). Haptipedia provides a comprehensive overview of grounded force-feedback devices (Seifi et al., 2019). Recent research in haptic technologies puts particular emphasis on cutaneous feedback, in part due to the lower complexity and lower cost of cutaneous feedback devices and their improved mobility (Prattichizzo et al., 2012).

Today, vibratory feedback is most widely used and most popular in commercial devices. The typical approach of binary on-off feedback or simple arrays to convey directional information (Meier et al., 2015) is arguably too restricted for rendering social touch. By controlling detailed parameters of vibration, such as amplitude and frequency, more expressive and nuanced output can be generated. Prior work has demonstrated various vibrotactile illusions, such as rendering directional cues (Culbertson et al., 2018), rendering the impression of compliance (Heo et al., 2019) or a specific surface texture (Strohmeier and Hornbæk, 2017; Strohmeier et al., 2020). While force mapping sensors are important to capture the nuances of touch (Kim et al., 2011), multi-modal sensors (Roberts et al., 2013) allow a strong personal and emotional way of interaction.

Feel-through tactile interfaces allow for new forms of tactile augmented reality, where real-world objects are superimposed with synthetic tactile renderings (Withana et al., 2018). Integrating touch sensors into deformable surfaces such as textiles (Orth et al., 1998), elastic polymers (Wessely et al., 2016; Teyssier et al., 2020) and even human skin (Weigel et al., 2015; Nittala et al., 2018) has enabled more expressive touch interaction. Emergent technologies are being developed to enrich digital social touch interactions and embed other input modalities such as force, shear, twist, and squeeze (Weigel et al., 2014). For example, tactile feedback can even be contactless and rendered in mid-air (Hoshi et al., 2010; Carter et al., 2013; Rakkolainen et al., 2021). Going beyond vibration (a point we make in the manifesto and return to later in this paper), skin deformation has been shown to add to the expressiveness and realism of haptic output. For instance, cutaneous haptic devices for the fingertip make use of skin indentation (Pacchierotti et al., 2014) or lateral stretching (Leonardis et al., 2015); haptic renderings with larger-scale skin deformation have been realized using adhesive skin patches with embedded artificial muscles (Hamdan et al., 2019). Moreover, flexible electronic skins are promising for conveying social touch; prior work covers vibratory (Yu et al., 2019) and electro-tactile (Withana et al., 2018) feedback amongst other modalities. It can be expected that advances in new materials (Biswas and Visell, 2021) and soft robotics will further expand the richness of multi-sensory haptic feedback (MacLean et al., 2017). In short, technological boundaries of what can be rendered, especially in cutaneous feedback, are continually being pushed. Novel actuators at different stages of maturity are in development that render a variety of tactile experiences (e.g., thermal feedback, pain, stickiness etc.) with potential for mediated digital social touch.

\section{Adoption of Haptic Technology: Haptic-Hype and Visions}

Against the rich backdrop of successful haptic devices outlined in the previous section, the integration of active haptic feedback (i.e., computer-controlled stimulation of the sense of touch via various actuators (Srinivasan and Basdogan, 1997; Hayward et al., 2004; Culbertson et al., 2018; Parisi, 2018)) in digital technology has been part of many visions on what interacting with these digital technologies should or could be like. Haptics has been envisioned as crucial to developing "the ultimate display" (Sutherland, 1965), has been described as vital for enabling "computing with feeling" interactions (Atkinson et al., 1977), has been called the "holy grail" of telepresence (Stone, 2000) or more recently, has been made central in the next generation of the Internet in the form of Tactile Internet (Fettweis, 2014). While many of these visions have been brought to full fruition others remain, for the time being, out-of-reach (Culberston et al., 2018; Parisi, 2018). Without diminishing the success of haptics as a field and within a variety of domains, there remains considerable debate on the claims made for haptic technology and what it can deliver in terms of social touch (Parisi et al., 2017; Parisi, 2018). Parisi (2018, p.32) argues that the promise of a haptic revolution in $\mathrm{HCI}$ is yet to be fulfilled: "[...]haptic interfaces are suspended in this state of perpetual immanence, always just on the horizon, always only five short years away [...]." Some have argued that haptic design tools (and their availability to interface designers) are too limited (Schneider et al., 2017), and that standardization of haptics is low in comparison to other modalities (Van Erp et al., 2010). Efforts are, however, underway within the industry to address these challenges. For example, Apple has recently formulated haptic design guidelines for developers creating applications for Apple products; Google has released guidelines for haptic design in Android applications; and manufacturers of haptic actuator technologies have united in a "Haptics Industry Forum" (https://hapticsif.org) to collectively address challenges in the field, including the standardization of "high definition" haptics. Other notable examples include MPEG which develops standards for coded representation including 
haptics (https://www.mpegstandards.org/standards/ Explorations/40/) and the Tactile Internet (https://standards. ieee.org/project/1918_1.html).

\section{MATERIALS AND METHODS}

As set out in the introduction the qualitative work presented in this article is explorative and uses an iterative process to get closer to and reflect upon (Srivastava and Hopwood, 2009; Aspers and Corte, 2019) the complexity of designers in relation to the social and sensorial aspects of designing digital social touch. This involved inductively building categories and themes to capture the participant collaborators' meanings to understand the "realworld" experiences, concepts, opinions, and challenges and opportunities that they face. This served to root and develop the manifesto in and through the interdisciplinary expertise of social touch designers/developers/researchers. The Manifesto for Digital Social Touch in Crisis was initiated at an Eurohaptics 2020 workshop titled "Designing Digital Touch: Social and Sensory Aspects and Challenges." The workshop itself was sparked by an interdisciplinary dialogue on touch between the social science team of the InTouch project (University College London, United Kingdom) and the computer science and engineeringoriented Interactive Skin project (Saarland University, Germany).

\section{Participants}

The workshop was targeted at researchers, interaction designers and developers interested in the challenges, methods and techniques of designing the social and sensorial aspects of digital social touch. The workshop call was disseminated and participants were recruited via relevant listings (e.g., ACM), a workshop website, and the Eurohaptics2020 conference. The 16 participants who attended the workshop have backgrounds in engineering, informatics, computer science, and $\mathrm{HCI}$, academia and industry and were based in France, Germany, United Kingdom, the Netherlands, Switzerland, India, China, and the United States. Ten participants (herein, they are referred to as participant collaborators) attended an optional follow-on collaborative workshop focused on the Manifesto, six of whom continued to contribute to the development of the manifesto (and are co-authors of this paper).

\section{Interdisciplinary Workshops}

The manifesto was developed over the next 5 months through a series of workshops, iterative feedback and revisions. Throughout the process Miro, a collaborative online platform was used to support, share and capture brainstorming, the outcome of activities, and discussion. The Miro boards provided data with which to map the process of the manifesto development, alongside facilitator notes, and group reflections on the process (Figure 2).

\section{Workshop 1: Designing Digital Social Touch}

Participant position papers, and $2 \mathrm{~min}$ introduction videos (stating their discipline, research focus and interest in the workshop) were shared prior to the workshop. Three $10 \mathrm{~min}$ "scene setting" talks were shared before the workshop: first, an overview of new haptic technologies and interfaces for skin (Steimle); second, a presentation of key issues concerning the sociality and sensoriality of digital social touch (Jewitt); and third, an introduction and demonstration of the "Multi-Touch Kit" (Pourjafarian et al., 2019), an open-source touch sensing toolkit. The workshop facilitated a group mapping activity (on Miro) to identify points of connection, contradiction and compatibility between these different perspectives on the designing digital social touch.

This collective map laid the groundwork for a second activity exploring the social and sensorial challenges of digitally mediating social touch using the Multi-touch toolkit, and the Designing Digital Touch Toolkit. These resources provided a grounding and springboard for the collective interrogation of digital social touch. Each group was facilitated by an interdisciplinary pairing (one socially orientated the other computer science/engineering orientated) and used design scenarios as prompts to elicit participants' experiences and to generate design considerations and key themes, challenges and opportunities for digital social touch design. The activities laid the groundwork for a "Manifesto" for digital social touch.

\section{Workshop 2: Manifesto}

The optional follow-on manifesto workshop comprised of preworkshop activities centered on understanding and exploring the manifesto form including working with the Manifesto Game (Ashby et al., 2019), and reviewing Workshop 1 Miro boards to suggest three to five themes for the manifesto. This helped to crystalize the issues and prioritize areas of the social and sensory design of digital social touch for the manifesto.

The workshop activities facilitated debate of the purpose of a manifesto for digital social touch, and collectively agreed a manifesto focusing statement. The rationale and starting point for "social touch in crisis" emerged from this debate. The consequences of different manifesto orientations, tones, openings, and provocations were explored. The right "feel" for a manifesto on digital social touch was agreed to be a challenging vision, playful, excited and hopeful, questioning and futurefacing, and provocative. The themes prioritized by participant collaborators were discussed, sifted, clustered and connections made across them to create higher-level themes. The interdisciplinary mix of participant collaborators, their cultural and political experiences, and immersion in different disciplinary and industry research/literature combined to produce a creative fusion on social touch and the digital. This was a lively process of debate that brought key concepts, challenges and opportunities to the fore and seeded a set of initial themes that were later developed into statements for the manifesto.

\section{Manifesto Development Process}

The Manifesto for Digital Social Touch in Crisis itself was developed through a collaborative and iterative cycle of review and revision. Initial themes, comments and ideas on the Miro boards were used to develop 12 draft manifesto statements: Manifesto Version 1. These were reformulated and consolidated into 10 statements in version 2, during which the workshop Miro boards were used to compile a complete collection of comments and ideas around each 
statement. These collections were used to compile a short paragraph elaborating on each manifesto statement, including some verbatim comments from the boards. Manifesto versions 2 and 3 were reviewed by the group, and revised to produce Manifesto Version 4 which was externally reviewed by six established international experts in haptics (collectively nominated by the authors) from $\mathrm{HCI}$, design, media and cultural studies, computer science, and engineering. They offered critiques of the manifesto form, questions of audience, terminology, definitions, language use (e.g., the exclusion of the word "human"), and highlighted statements or aspects of digital social touch (e.g., privacy) that they considered required more development or emphasis. The reviewers' feedback and a final review by participant collaborators informed the final manifesto (version 5) at the center of this paper.

\section{FINDINGS: CURATING THE MANIFESTO STATEMENTS}

In this section, we make the history of the manifesto statements visible by describing the process of how the statements were curated, reordered, clustered and consolidated to realize the shape and flow of the final manifesto. The quotes used are written comments on the Miro boards or, if indicated, from the expert reviewer comments.

Throughout, a key consideration was getting the right tone for the manifesto statements through the collective iteration and development of the heading and content of each statement. For example, statement 2 was initially titled "Balance Touch and Technology" and as the manifesto developed, participant collaborators felt that title was not strong enough: "This could use a more provoking title, the priority is the experience of touch, technology is just a tool to achieve it." It was amended to "Touch first, technology second." Conversely, for Statement 5 the original formulation of "Lose Vibration! Feel Beyond the Habituated" was later considered to be too strong, and was changed to read, "Move beyond vibration: Feel beyond the habituated." Similar remarks were made about the content of other manifesto statements. For example, the text for Statement 7 "Remake, Don't Only Replicate!" included the statement "Trash the touch-screen," which a participant collaborator noted, "I would perhaps add "devoid of feedback" or something like that, as touchscreens per say in HCI have opened up many avenues in education, accessibility to information, etc.," a suggestion that informed the iteration of the manifesto.

A key aspect of the external review was the sense of how the manifesto might "land" within the research field. The manifesto format was quite new and somewhat confounding for some: “... the form of writing serves another purpose than having a scientific discussion about what we know and which facts we know about touch". At the same time, however, the manifesto was found stimulating, and the reviewers were positive about the format: "The style of the manifesto is very new to me and although initially confusing (just the way it is written and words used, syntax and semantics), I see how it can draw in attention"; "I found it highly inspiring, not only in its content but also in the structure; I felt it was controlling my train of thoughts, in creative ways."
These iterative cycles of review, comment and revision led to the Manifesto for Digital Social Touch in Crisis presented in full at the beginning of this paper Figure 1, each statement is presented and discussed below.

\section{Statement 1: Make Social Touch Central}

This statement (initially named - Keep touch central) reflects the collective view that there is a need to bring an urgent emphasis to social touch, linking to the significance of the social aspects of touch interactions (set out in the background literature), for wellbeing and communication (Gallace and Spence, 2010; McGlone et al., 2014) and development (Cascio et al., 2019) and how these have inspired investigations into the use of haptic technology for social touch (Huisman, 2017).

One expert reviewer noted that "the word "Human" is mentioned only once, for "non-human." In my opinion, it is quite central and should be mentioned." In response, "human" was included in the text of the statement. There was a broader sense of the need to ensure that social touch - not only its technical realisation - remains "center stage," both in the context of visual and audio prominence in digital spaces and the Covid-19 pandemic. The "at risk" sense arose from the notion of "poor" social touch interactions due to the way technology is designed, for example the prominence of flat screens, or "minimized" touch effects such as the buzzing of smartphones (Culberston et al., 2018; Parisi, 2018). The statement also reflects the priority that is generally given to visual and auditory modalities, while the tactile is more difficult to realistically achieve in digital social touch devices accessible to the public (due to cost, form factors, functions/roles, etc., so in turn limited to buzzing), and as a result, is perceived as superfluous or not as convincingly necessary as audio or vision.

\section{Statement 2: Design Touch First, Technology Second}

This statement combines what were originally (in earlier versions of the manifesto) two statements - Marginalize the technology and Amplify interdisciplinarity. This highlights the need to foster the richness of haptics set out in the background of this article, and to encourage the ongoing development or realization of "better" digital social touch, and the value of interdisciplinarity (Jewitt et al., 2020). Marginalize the technology related to a sense that technology dominates the haptics scene (e.g., conferences, papers, projects), shaping digital social touch in particular ways and aimed to argue for less technology-driven development: it generated significant debate among the participant collaborators. The intention was to emphasize that technology should not be the (sole) driving force, however, while recognizing the need to reduce the dominance of technology, "marginalize" was considered too strong a term, since technology is important. As one participant collaborator wrote, "Right now technology seems to be driving development of applications and user experiences involving digital touch, but at its core technology is supposed to be a tool to achieve a goal, and maybe we want that goal to be set by something else than just the availability of certain techs?" Rather than a "techno-push" the manifesto also points to a 
need to ensure users are included in the design process and remain at the core of the technology. Alternative statement suggestions included "Touch first, tech second," and "Avoid technology dominance." An expert reviewer with a design background liked this statement: "This is my favorite! I always use this as the only teaching rule for my wearable technologies course. Design experience first, technology second. I think it's great!." The other statement Amplify interdisciplinarity that was folded into this final manifesto statement, again to mark the insufficient attention to the creative arts and sought to redress the dominance of Computer Science and Engineering in the development of touch technology. Promoting interdisciplinarity was considered one effective way to avoid technology dominance.

\section{Statement 3: Democratize Touch: Don't Lock It In}

This statement links with the discussion of social touch as a cross-cultural (Carra et al., 2014) global phenomenon (Sorokowska et al., 2021) in the background literature section. It was also inspired by Lanier's description (Lanier., 2010, p7-9) of "lock-in" as a process which causes "digital designs to get frozen into place...culling the ambiguities of flexible thoughts as more and more thought structures are solidified into effectively permanent reality." The statement went through a variety of title changes - Democratize touch and later, "Don't lock touch in: desire its heterogeneity," in an effort to highlight the potential role people can play in the creation and definition of touch. It involved much discussion: "...when we say everyone should be involved, do we then mean everyone in the field or also the average user?." The consensus was that democratization meant involving all people - "if you have a sense of touch, you are pretty much automatically qualified to contribute to defining what digital touch can become." This statement had social and political connotations, raising many questions for participant collaborators including who drives the norms around touch? How do we make sure we all have a say in how touch gets "created"? How do we address power imbalances? While for others the statement arose from the need for touch to be designed such that end-users would have the agency to personally define and develop their own touch language.

Ultimately, this manifesto statement speaks to a need to develop "opportunity spaces" that support exchange between users and developers and designers, prior to "creator" notions of touch, social touch and digital social touch get "locked-in." Touch cultures were seen to be at risk from the (digital) touch norms that were established by one culture developing the technologies of digital touch: notably the risk of hegemonic companies "flattening" culture and individuality. However, there was also a recognition of diversity needing to be brought into balance with notions of digital social touch design standardization (Van Erp et al., 2010). This statement highlights the need to keep cultural distinctions or even to amplify the (cultural) diversity of touch that exists (Remland et al., 1991). As one participant collaborator noted, "Touch is a conversation and like a conversation it should be open to develop and is made by interaction."

\section{Statement 4: Protect Touch: Keep Touch Private and Secure by Design}

This statement speaks to critical concerns of privacy, security and ownership of touch communications, such as maintaining ownership and share-ability rights over one's touch "record and replay." These concerns run like a thick seam through the strata of all technologies (Kamleitner and Mitchell, 2019), are never far from the surface of haptics, and were consistently raised as important throughout the process of developing the manifesto. That process brought out different, although related specificities, highlighting the complexity of privacy of touch. For example, concerns were expressed regarding the need to account for privacy "when you start recording and sharing touch gestures" and the need to "protect touch ownership" and guarantee control over privacy with participant collaborators suggesting they would want "control over what information about me and my way of interacting through touch becomes public or not" as well as "what touches me, how it touches me, and what information is conveyed to me through touch." There was agreement that touch data, "perhaps even more than other types of data given the connotations with intimacy" needed to be protected. Similar to issues raised about how users can be identified through their body posture/motions this could also apply to touch (see Miller et al. (2020) for an example in the context of VR; Ornati., 2022. A comment by one of the expert reviewers raised the phrase "Keep touch private and secure by design" from the explanatory text to the statement heading. While another expert reviewer comment helped us to reshape the manifesto framing of touch ownership: "It starts too negative, when this ownership is actually so liberating and empowering. Would there be scope to start with something on the flip side of this ownership? E.g.: Touch is intimate. Touch reveals myself and my boundaries. Touch can be intrusive." This helped us to rewrite the opening paragraph of this manifesto statement.

\section{Statement 5: Move Beyond Vibration: Feel Beyond the Habituated}

This statement emerged in response to the dominance of vibration and the emergence of new actuators for social touch (see the background section), as well as participant collaborators' express desire to disrupt technological interfaces (e.g., through notions of "touchless touch" (mid-air haptics) or extending touch beyond the hand to a whole body experience) that create a reduction of sensation in types of touch, touch feedback (e.g., primarily vibration motors) or touch experiences. It was agreed in order to bring the richness of "real" touch into the digital, that there is a need to move out of the restricted range of both actuation technologies and interface materials that are available in current digital social touch implementations. A variety of possible formulations of this emerged: "Move beyond vibration and glass," "Move beyond flat, move beyond vibe," "Move beyond a rigid surface," or "Touch beyond vibration." However, we recognized the need not to assume 
touch research is only about flat surfaces and vibrations, and to include work on grounded force-feedback (Seifi et al., 2019) and skin deformation (Hamdan et al., 2019). (For further examples, see also Tibbits (2017)). This consideration led to the final manifesto statement.

\section{Statement 6: Foster Exploration of Meaningful Touch Experiences}

This statement (initially named "Create responsive touch experiences") focuses on personalizing touch to enable touch to be responsive to the individual (touch preferences) and context. This statement links to the need for digital social touch to respond to on touch cultures, the variation and levels of intimacy of social touch, and the importance of embedding digital touch into social sensory contexts of established social touch communication - all of which are complex issues that raise questions for the design of effective mediated social touch (see Background section). Discussion of this statement involved suggestions of using the term reciprocal to be more explicit that touch interaction concerns bi-directional communication rather than being a technical property. Participant collaborators considered an emphasis on the personal to be critical, or the adaptive which captures both reciprocity and personalization. The contextual needs of touch experience were foregrounded, such as in mobility, static, outdoors vs. indoors. The adaptability to context was also highlighted, particularly in terms of privacy, with possible noise or simulations generated by touch technologies. These aspects are encompassed in the "meaningfulness" of touch experience.

\section{Statement 7: Remake, Don't Only Replicate!}

This statement - originally named Stop replicating touch and later Don't just replicate touch, remake it, highlights a tension in the design and development of social touch between a drive to replicate or mimic touch (Price et al., 2021) versus designing new forms of touch experiences (see Background). It is aimed at encouraging a move beyond replication to forms of more innovative and creative digital social touch experiences. Participant collaborators noted that replicating is, however, sometimes needed or desired and saw the real issue as being that digital social touch should not be limited to replication: "...remaking is making anew, which I tend to associate with technology. So perhaps it should be made clear in the explanation that it is not only about technologizing touch in yet to be imagined spaces." The statement is an offer of inspiration and an invitation to think outside the box.

\section{Statement 8: Manage Great "Tech-Xpectations"}

This statement concerns the need to manage both user expectations of technology in relation to commercial hype and marketing and the vision and promise of digital social touch (Parisi et al., 2017; Parisi, 2018) (see Background). It is informed by discussion of how personal experience can lead to predictions of how "digital social touch" should feel, and result in disappointment or surprise if/when the actual feeling is different. Suggestions involved offering clarity on what is technologically possible, and both the lowering of expectations by avoiding overly hyperbolic advertising and the changing of user expectations through education and communication on new forms of haptic feedback. Touch technologies were, it was argued, a long-term endeavor. The statement was seen as "offering a user counterpart to statement 9" and an invitation to designer/developers "to try crazy new things (i.e., don't worry, go try these new ideas, the digital touch you are designing should feel different from what's already out there/ what people expect)." One collaborator noted that in French law (Republic Francaise, 1994), if a photograph is used in the media is retouched it must declare that it is "photo retouchée" and they suggested that the same could be required of touch, as in "toucher retouchée" as a way to temper the hype of digital social touch promises and to reframe expectations.

\section{Statement 9: Develop Open Touchy Tools}

This statement focuses on the need for new design tools for the development of touch experiences that expand our perception of touch and touch vocabularies. Participant collaborators agreed that a diverse range of technical tools is needed to enable designers to include touch more broadly (Schneider et al., 2017; Seifi et al., 2020), and to help broaden thinking about touch: "to build shared resources for designing and talking about touch." For example, design tools to engage with the social and sensory aspects of touch (e.g., Designing Digital Touch Toolkit https://www.in-touch-ucl.design). The statement focuses both on tools and the need to share these (discussed in the Background section), as one participant collaborator noted, "it is also about making it accessible to anyone or with various levels (public, tech experts, designers), in transparency of the device(s) used, with adaptation to the context of use (mobile phone, VR, etc.)." The significance of access was incorporated in the final manifesto statement, as was the need for tools to be "open" to resonate with design and engineering communities.

\section{Statement 10: Keep Speculating}

This final manifesto statement was motivated by a need to draw attention to wider social and political responsibilities for technology development, particularly given the lack of social forecasts for touch, and the often-unintended negative consequences of existing applications. Some highlighted the need to outline future utopian and dystopian scenarios for digital social touch. The statement can be read in two ways: first, "keep speculating" in terms of imagining digital social touch; and second, a wider political use of the term speculation in relation to a lack of regulation and social responsibility in which manifestos are seen as calling "for political or judicial reconfigurations" rather than placing such responsibility for action on designers and developers (informed by Fritsch et al.'s (2018) analysis of IoT manifestos). This statement is also a call to users, decision-makers and regulators to consider how to develop and regulate digital social touch design in its nascent stages, to be imaginative, and to actively think through future scenarios and potential consequences as part of the design process. The call to speculation is aimed at energizing the field to: "Move beyond the Feelies" (Huxley, 1932)! 
The above 10 statements comprise the Manifesto for Digital Social Touch in Crisis.

\section{DISCUSSION}

Through analysis and reflection on the process of developing the manifesto, opportunities and challenges, and key themes were identified as foundational to engaging with the social and sensory aspects of designing digital social touch. These informed the manifesto development. Each is discussed below (the quotes used are written comments on the Miro boards).

\section{Opportunities and Challenges}

Throughout the development of the manifesto, digital social touch was considered to offer growing opportunities in terms of bringing new awareness to the importance of touch and haptics more broadly. The global Covid-19 pandemic was understood to have increased awareness of the value and importance of touch communication (particularly in contexts of social isolation) and prompted people to question their touch needs. A need for digital social touch and an appreciation of the way it can provide a sense of closeness when apart, particularly for those who cannot leave their homes was seen as an opportunity. One consequence of this is a new context for understanding the design of digital social touch. The potentials of technology were seen to offer opportunities for new ways to develop remote touch, from integrating touch functionality "into virtually any surface and material," to haptic illusions (Hayward, 2008), or robotic skin and autonomous systems that can sense touch. Digital social touch was felt to open up new and different ways of engaging, such as through physical interaction not possible with "analogue" touch (e.g., replay touch or record/saving of tactile memories), generating software frameworks for prototyping and implementing different haptic interfaces or parameterizing haptic design for people from other fields to design digital social touch.

Emerging challenges for engagement with the social and sensory aspects of digital social touch through design were also identified.

The conceptualization of touch, that is, how researchers, designers, engineers, or lay people conceptualize touch was a challenge in the development of digital social touch. People's general understanding of touch was perceived as being limited oftentimes to ritualized human-human touches (e.g., handshakes). This serves to foreground touch in terms of functional rather than social encounters or touch as utilitarian as opposed to aesthetic (e.g., touch that is pleasurable). It also excludes touch with other entities as one participant collaborator noted, "I feel I have a rich language of touch with my cats but this seems to be "forgotten" when we think about touch tech." This prompted conversation around mimicking or replicating touch. Should we or can we mimic social touch through technology? Challenges around which features of touch were important and how to manage these, were linked to the ways that touch was conceptualized (Price et al., 2021). For example, how to manage sensory input and output or how to integrate contextual factors surrounding touch. Of particular prominence were notions of control: how to allow options of control (e.g., touch on which body parts, and the right to be unavailable), finding haptic encodings that are comprehensible and acceptable to users across different genders and cultures, and how long to keep a recording of a digital social touch, similar to the "right to be forgotten" (https://gdpr-info.eu/art-17-gdpr/).

Several technical challenges were identified, including how to identify the most appropriate interface for a specific social touch experience or end-user. Other challenges centered on the early development of digital social touch design. For example, content design tools for haptic sensations whilst recognised through some significant commercial successes (see Background), were nonetheless considered less mature than visual and auditory modalities, which somewhat limits possibilities for people in non-technical fields to creatively explore the medium. Further considerations concerned knowing what is and is not "designable" and a lack of extensive data sets for a wide range of "real haptic stimuli."

Three methodological challenges in the design of digital social touch were discussed. First, the challenge of how to undertake sensory measurements to collect data on people's touch sensations at scale, that is to build libraries of different kinds of sensory feedback around the body, including the measurement of people's thresholds for negative and positive touch, and the development of standards or benchmarks for digital social touch. Second, a need for more interaction design tools for digital social touch. These included a need for a common language of touch (developing libraries) and developing metaphors for design; recognizing the different affordances of haptic technologies for digital social touch. Third, challenges were raised related to a need the design of digital social touch interfaces to support end-user customization; the development of software frameworks for prototyping and implementing interfaces to support creatively "playing with" digital social touch concepts and foster design. Interdisciplinarity was felt to be key to overcoming these challenges. Collaborations that brought together "engineering, material, experience designers, and social scientists (and others) from an early stage" and built "relationships across academia and industry" were valued as highlighting the importance of developing "A shared vocabulary when describing touch (users, designers, researchers)." This was seen as valuable to "Avoid techno-push attitude: more communication or steps to opening minds on disciplinary collaborations/users at the center, not solely the beauty of the technology."

A number of socially orientated challenges emerged in relation to user expectations and the digital translation of aspects of social touch. The management of user expectations drew attention to the need for the design of digital social touch to consider users' prior social touch experiences and how this shapes expectation. As one participant collaborator put it, "Users have personal experience and might predict that the "digital touch" feels a certain way and be disappointed/surprised when the actual feeling is different from this expectation." The notion of branding was highlighted as needing careful consideration, particularly in terms of what brands convey, and how they manage users' "fears" of digital social touch, and whether and how people "should" be encouraged to overcome such concerns. 
This speaks to a tension between users' generally high expectations of technology versus what is realistically possible with current touch technologies, and a need to balance these two factors to avoid user disappointment or manage surprise when tactile sensations differ from prior touch experiences. Relatedly, the challenge of individual differences in people's touch experiences was raised as a challenge, with the recognition that touch is socially and culturally bound and idiosyncratic to some extent, raising the challenge of how these experiences could be responded to from a "fixed" engineering or design perspective. Linked to this point, the question of how to frame digital social touch, which can allow us to touch differently from the "physical" world, was seen as significant in fostering users' responses to it: from an open-acceptance versus evoking fear. This links directly to models of the commercialization, branding and business that digital social touch is embedded within, and raises the challenge of how to avoid haptic-monitoring and control of haptic content (likened to Facebook type models) and/or the use of haptic data as yet another source of data for building user-profiles for the targeting of individuals (e.g., for advertising).

\section{Key Themes}

The need to enrich digital social touch experience: the need to move beyond vibrotactile applications and the status-quo of touch to make digital social touch a richer experience was consistently expressed. "Status-quo is touch in the form of touch screens. A way of thinking perpetuated by tech companies selling devices + apps. Thinking about touch seems mostly visual in this situation (also in the addition of touch to $A R / V R$ ). How do we break this way of thinking?' It was suggested that in order to do this there is a need to rethink what digital social touch is, what it means, and could be. That is, participant collaborators felt the need to extend beyond realism and known experiences of touch to consider and design new forms of touch (whilst balancing user expectations and acceptability): to extend haptic technology beyond mimicking human tactile experiences. Some participant collaborators questioned whether it is possible to mimic social touch through technology. Ultimately there was a sense that rethinking digital social touch demands a more nuanced understanding of touch, whether by collecting people's touch sensations at scale, understanding individual and cultural touch needs and perceptions, or identifying and mapping the nuances of touch and reviewing potential use-cases for digital social touch.

The need to engage with the wider socio-political context of touch: Social distancing practices resulting from responses to the Covid-19 pandemic newly underscored the need for digital social touch and prompted discussion of the political character of touch and physical proximity. As one participant collaborator noted, "I'm also reminded of how COVID strikes those in poorer communities harder because they do not have the opportunity for social distancing. In a way their physical proximity/touch practices are determined by their socio-economic status. How do we see touch technologies when taking these aspects into consideration?." The "medium" of touch (i.e., the skin) and the question of who gets to touch what and who and when was considered a significant theme.

The design of touch privacy spoke to issues of consent, control, and ultimately human agency. The debate of these issues involved consideration of how designers and developers can incorporate consent into touch technology. This raised questions of when and where the user (including the receiver of a touch message) is in control, with the right to be unavailable, and manage monitoring or privacy of communication. Participant collaborators concluded that digital social touch needs to develop acceptable haptic encodings, as noted from the discussion (and on stickies) "that are comprehensible and acceptable for different users (gender, culture etc.)" and which foster a sense of user agency in giving and receiving digital social touch.

Drawing on these opportunities, challenges and themes, the manifesto for digital social touch in crisis aims to provoke and prompt new ideas by opening an interdisciplinary space to question and imagine newly digital social touch. The manifesto seeks to be a bridge between HCI, computer science and engineers, and social scientists engaged with digital social touch - to "give new life to" (Hanna et al., 2019:2) the design of digital social touch.

\section{CONCLUSION}

This paper has offered a provocative call to action to designers, developers and researchers to engage more deeply with the social and sensory aspects of digital social touch in the form of the Manifesto for Digital Social Touch in Crisis. Drawing on the research literature and analysis of data from this interdisciplinary collaboration - centered on a series of workshops and collective iteration of the manifesto, the paper has highlighted the key opportunities, challenges and central themes that provided foundational steps in the development of the manifesto. These included the growing opportunities for touch offered by technologies, and increased awareness of the significance of social touch, the technical, methodological, and socially orientated challenges of designing digital social touch, including the development of design tools to enrich digital social touch experience, and engage with the wider socio-political context of touch. The paper has made visible the collective iterative process of curating, clustering and consolidating the manifesto statements, making the process transparent and signaled the potential of placing the social and sensory aspects of touch at the centre of the design and development of digital social touch.

As society engages with and emerges from the uncertainty of touch in Covid-19 times, the Manifesto for Digital Social Touch in Crisis signals a desire for change and offers a set of 10 provocations to support a rethinking and reimaging of the social and sensory aspects of touch through the design process. The ten manifesto statements offer designers, developers and researchers across different disciplines routes toward future roadmaps or directions for digital social touch in society.

\section{DATA AVAILABILITY STATEMENT}

The datasets presented in this article are not made publicly available because the identity of workshop participants/data (Miro boards) is linked to specific statements and participant log-in info in Miro and it is not possible to anonymise that data. Further queries regarding the datasets should be directed to $c$. jewitt@ucl.ac.uk. 


\section{ETHICS STATEMENT}

The studies involving human participants were reviewed and approved by IOE, University College London ethics board. The patients/participants provided their written informed consent to participate in this study.

\section{AUTHOR CONTRIBUTIONS}

$\mathrm{CJ}$, SP, and JS contributed to the conception and design of the study. CJ, SP, JS, LG, NP, and GH organized the research workshops. CJ and SP and GH performed the qualitative analysis of the study data. CJ led the drafting and writing of the manuscript. SP, JS, and GH wrote sections of the manuscript. All authors contributed to the development of ideas, manuscript revision, read, and approved the submitted version.

\section{REFERENCES}

Ardiel, E. L., and Rankin, C. H. (2010). The Importance of Touch in Development. Pediatr. child Health 15 (3), 153-156. doi:10.1093/pch/15.3.153

Ashby, S., Hanna, J., Matos, S., Nash, C., and Faria, A. (2019). Fourth-Wave HCI Meets the 21st Century Manifesto: Proceedings of the Halfway to the Future Symposium 2019 Article 23 Pages 1-11. doi:10.1145/3363384.3363467

Askari, S. I., Haans, A., Bos, P., Eggink, M., Lu, E. M., Kwong, F., and IJsselsteijn, W. (2020). "Context Matters: The Effect of Textual Tone on the Evaluation of Mediated Social Touch," in International Conference on Human Haptic Sensing and Touch Enabled Computer Applications, Cham (Springer), 131-139. doi:10.1007/978-3-030-58147-3_15

Aspers, P., and Corte, U. (2019). What Is Qualitative in Qualitative Research. Qual. Sociol. 42 (2), 139-160. doi:10.1007/s11133-019-9413-7

Atkinson, W. D., Bond, K. E., Tribble, G. L., III, and Wilson, K. R. (1977). Computing with Feeling. Comput. Graphics 2 (2), 97-103. doi:10.1016/00978493(77)90009-7

Bales, K. L., Witczak, L. R., Simmons, T. C., Savidge, L. E., Rothwell, E. S., Rogers, F. D., et al. (2018). Social Touch during Development: Long-Term Effects on Brain and Behavior. Neurosci. Biobehavioral Rev. 95, 202-219. doi:10.1016/ j.neubiorev.2018.09.019

Beßler, R., Bendas, J., Sailer, U., and Croy, I. (2020). The "Longing for Interpersonal Touch Picture Questionnaire": Development of a New Measurement for Touch Perception. Int. J. Psychol. 55 (3), 446-455. doi:10.1002/ijop.12616

Bickmore, T. W., Fernando, R., Ring, L., and Schulman, D. (2010). Empathic Touch by Relational Agents. IEEE Trans. Affective Comput. 1 (1), 60-71. doi:10.1109/ T-AFFC.2010.4

Biswas, S., and Visell, Y. (2021). Haptic Perception, Mechanics, and Material Technologies for Virtual Reality. Adv. Funct. Mater. 31, 2008186. doi:10.1002/ adfm.202008186

Block, A. E., Christen, S., Gassert, R., Hilliges, O., and Kuchenbecker, K. J. (2021). "The Six Hug Commandments," in Proceedings of the 2021 ACM/IEEE International Conference on Human-Robot Interaction, 380-388. doi:10.1145/3434073.3444656

Breitschaft, S. J., Clarke, S., and Carbon, C.-C. (2019). A Theoretical Framework of Haptic Processing in Automotive User Interfaces and its Implications on Design and Engineering. Front. Psychol. 10, 1470. doi:10.3389/ fpsyg.2019.01470

Bull, M., Gilroy, P., Howes, D., and Kahn, D. (2006). Introducing Sensory Studies. Senses Soc. 1, 1, 2006 (Mar. 2006), 5-7. doi:10.2752/ 174589206778055655

Cang, X. L., Bucci, P., Strang, A. J., Allen, J., MacLean, K. E., and Liu, H. Y. S. (2015). "Different Strokes and Different Folks: Economical Dynamic Surface Sensing and Affect-Related Touch Recognition," in Proceedings of the 2015 ACM on

\section{FUNDING}

This work was supported by the European Research Council (ERC) under the European Union's Horizon 2020 research and innovation programme: In-Touch (PI Jewitt, Consolidator Grant agreement No. 681489) and Interactive Skin (PI Steimle, Starter Grant agreement No. 714797).

\section{ACKNOWLEDGMENTS}

We would like to thank all of the participants who participated in the workshop. Special thanks to Dasha Kolesnyk, Elena Stoll, Salma Thalji, and Douglas Atkinson who contributed to the development of the Manifesto. We are grateful to the six expert reviewers who critically read and commented on an earlier draft of the Manifesto.

International Conference on Multimodal Interaction (ICMI '15), Seattle, WA, USA (New York, USA: ACM), 147-154.

Carra, C., Lavelli, M., and Keller, H. (2014). Differences in Practices of Body Stimulation during the First 3 Months: Ethnotheories and Behaviors of Italian Mothers and West African Immigrant Mothers. Infant Behav. Dev. 37 (1), 5-15. doi:10.1016/j.infbeh.2013.10.004

Carter, T., Seah, S. A., Long, B., Drinkwater, B., and Subramanian, S. (2013). "UltraHaptics," in Proceedings of the 26th annual ACM symposium on User interface software and technology (New York, USA: UIST '13), 505-514. doi:10.1145/2501988.2502018

Cascio, C. J., Moore, D., and McGlone, F. (2019). Social Touch and Human Development. Dev. Cogn. Neurosci. 35, 5-11. doi:10.1016/j.dcn.2018.04.009

Caws, M. (2001). Manifesto. A Century of Isms. Lincoln: University of Nebraska Press. $\mathrm{X}$-xxxi.

Chopik, W. J., Edelstein, R. S., van Anders, S. M., Wardecker, B. M., Shipman, E. L., and Samples-Steele, C. R. (2014). Too Close for comfort? Adult Attachment and Cuddling in Romantic and Parent-Child Relationships. Personal. Individual Differences 69, 212-216. doi:10.1016/j.paid.2014.05.035

Crusco, A. H., and Wetzel, C. G. (1984). The Midas Touch. Pers Soc. Psychol. Bull. 10 (4), 512-517. doi:10.1177/0146167284104003

Culbertson, H., Schorr, S. B., and Okamura, A. M. (2018). Haptics: The Present and Future of Artificial Touch Sensation. Annu. Rev. Control. Robot. Auton. Syst. 1, 385-409. doi:10.1146/annurev-control-060117-105043

Danieau, F., Fleureau, J., Cabec, A., Kerbiriou, P., Guillotel, P., Mollet, N., Christie, M., and Lécuyer, A. (2012). "Framework for Enhancing Video Viewing Experience with Haptic Effects of Motion," in Proceedings of the IEEE Haptics Symposium (IEEE), 541-546. doi:10.1109/haptic.2012.6183844

Debrot, A., Schoebi, D., Perrez, M., and Horn, A. B. (2013). Touch as an Interpersonal Emotion Regulation Process in Couples' Daily Lives. Pers Soc. Psychol. Bull. 39 (10), 1373-1385. doi:10.1177/0146167213497592

den Dekker, A., Dima, L. A., Huisman, G., and Shor, D. (2021). “July)Future Affair: An Artistic Exploration of Socially Distanced Affective Touch via Forearm Stimulation," in 2021 IEEE World Haptics Conference (WHC) (IEEE), 877.

Ditzen, B., Neumann, I. D., Bodenmann, G., von Dawans, B., Turner, R. A., Ehlert, U., et al. (2007). Effects of Different Kinds of Couple Interaction on Cortisol and Heart Rate Responses to Stress in Women. Psychoneuroendocrinology 32 (5), 565-574. doi:10.1016/j.psyneuen.2007.03.011

Döring, N., and Pöschl, S. (2018). Sex Toys, Sex Dolls, Sex Robots: Our Underresearched Bed-Fellows. Sexologies 27 (3), e51-e55. doi:10.1016/j.sexol.2018.05.009

Durkin, J., Jackson, D., and Usher, K. (2021). Touch in Times of COVID-19: Touch Hunger Hurts. J. Clin. Nurs. 30. doi:10.1111/jocn.15488

Eid, M. A., and Al Osman, H. (2016). Affective Haptics: Current Research and Future Directions. IEEE Access 4, 26-40. doi:10.1109/ACCESS.2015.2497316

Elsden, C., Speed, C., Nissen, B., Murray-Rust, D., and Tallyn, E. (2019). Four Manifestos from 'HCI for Blockchain' Ubiquity. The J. Pervasive Media 6 (4), 27-30. Number 1, November 2019. doi:10.1386/ubiq_00004_7 
Erk, S. M., Toet, A., and Van Erp, J. B. F. (2015). Effects of Mediated Social Touch on Affective Experiences and Trust. PeerJ 3, e1297. doi:10.7717/peerj.1297

Fettweis, G. P. (2014). The Tactile Internet: Applications and Challenges. IEEE Veh. Technol. Mag. 9 (1), 64-70. doi:10.1109/MVT.2013.2295069

Field, T. (2014). Touch. 2nd Ed. Cambridge, Massachusetts, US: The MIT Press.

Field, T. (2002). Violence and Touch Deprivation in Adolescents. Adolescence 37 (148), 735-749.

Field, T., Poling, S., Mines, S., Bendell, D., and Veazey, C. (2020). Touch Deprivation and Exercise during the COVID-19 Lockdown April 2020. Mraj 8 (8). doi:10.18103/mra.v8i8.2204

Field, T. (2019). Social Touch, CT Touch and Massage Therapy: A Narrative Review. Dev. Rev. 51, 123-145. doi:10.1016/j.dr.2019.01.002

Field, T. (2010). Touch for Socioemotional and Physical Well-Being: A Review. Dev. Rev. 30 (4), 367-383. doi:10.1016/j.dr.2011.01.001

Fotopoulou, A., and Tsakiris, M. (2017). Mentalizing Homeostasis: The Social Origins of Interoceptive Inference. Neuropsychoanalysis 19 (1), 3-28. doi:10.1080/15294145.2017.1294031

Fritsch, E., Shklovski, I., and Douglas-Jones, R. (2018). Calling for a Revolution: An Analysis of IoT Manifestos CHI 2018. MontrealCanada,: QCACM. 978-1-45035620-6/18/04. doi:10.1145/3173574.3173876

Gallace, A., and Spence, C. (2010). The Science of Interpersonal Touch: An Overview. Neurosci. Biobehavioral Rev. 34 (2), 246-259. Feb. 2010. doi:10.1016/j.neubiorev.2008.10.004

Goldstein, P., Shamay-Tsoory, S. G., Yellinek, S., and Weissman-Fogel, I. (2016). Empathy Predicts an Experimental Pain Reduction during Touch. The J. Pain 17 (10), 1049-1057. doi:10.1016/j.jpain.2016.06.007

Green, L., and Moran, L. (2021). Covid-19, Social Distancing and the 'scientisation' of Touch: Exploring the Changing Social and Emotional Contexts of Touch and Their Implications for Social Work. Qual. Soc. Work 20 (1-2), 171-178. doi:10.1177/1473325020973321

Green, L. (2017). The Trouble with Touch? New Insights and Observations on Touch for Social Work and Social Care. Br. J. Soc. Work 47 (3), bcw071-792. doi:10.1093/bjsw/bcw071

Guerrero, L. K., and Andersen, P. A. (1991). The Waxing and Waning of Relational Intimacy: Touch as a Function of Relational Stage, Gender and Touch Avoidance. J. Soc. Personal Relationships 8 (2), 147-165. doi:10.1177/ 0265407591082001

Haans, A., de Bruijn, R., and IJsselsteijn, W. A. (2014). A Virtual Midas Touch? Touch, Compliance, and Confederate Bias in Mediated Communication. J. Nonverbal Behav. 38 (3), 301-311. doi:10.1007/s10919-014-0184-2

Haans, A., and IJsselsteijn, W. A. (2009). The Virtual Midas Touch: Helping Behavior after a Mediated Social Touch. IEEE Trans. Haptics 2 (3), 136-140. doi:10.1109/TOH.2009.20

Haans, A., and IJsselsteijn, W. (2006). Mediated Social Touch: a Review of Current Research and Future Directions. Virtual Reality 9 (2), 149-159. Mar. 2006. doi:10.1007/s10055-005-0014-2

Halley, J. (2007). Boundaries of Touch: Parenting and Child Intimacy. Champaign, IL: University of Illinois Press.

Hamdan, N. A.-h., Wagner, A., Voelker, S., Steimle, J., and Borchers, J. (2019). "Springlets," in Proceedings of the 2019 CHI conference on human factors in computing systems. doi:10.1145/3290605.3300718

Hanna, J., Ashby, S., Matos, S., Faria, A., and Rodrigues, R. (2019). "Dissent by Design," in CHI Conference on Human Factors in Computing Systems Extended Abstracts (CHI'19 Extended Abstracts), Glasgow, Scotland UKNew York, NY, USA, May 4-9, 2019 (New York, USA: ACM), 10. doi:10.1145/3290607.3310423

Hannaford, B., and Okamura, A. M. (2016). "Haptics," in Springer Handbook of Robotics (Cham: Springer), 1063-1084. doi:10.1007/978-3-31932552-1_42

Harrington, K., Large, D. R., Burnett, G., and Georgiou, O. (2018). "Exploring the Use of Mid-air Ultrasonic Feedback to Enhance Automotive User Interfaces," in Proceedings of the 10th International Conference on Automotive User Interfaces and Interactive Vehicular Applications (AutomotiveUI '18), New York, NY, USA (New York, USA: Association for Computing Machinery), 11-20. doi:10.1145/3239060.3239089

Hayward, V. (2008). A Brief Taxonomy of Tactile Illusions and Demonstrations that Can Be Done in a Hardware Store. Brain Res. Bull. 75 (6), 742-752. doi:10.1016/j.brainresbull.2008.01.008
Heo, S., Lee, J., and Wigdor, D. (2019). "PseudoBend: Producing Haptic Illusions of Stretching, Bending, and Twisting Using Grain Vibrations," in Proceedings of the 32nd Annual ACM Symposium on User Interface Software and Technology (New York, USA: UIST '19), 803-813. doi:10.1145/3332165.3347941

Hertenstein, M. J., Holmes, R., McCullough, M., and Keltner, D. (2009). The Communication of Emotion via Touch. Emotion 9, 566-573. 4 (2009). doi:10.1037/a0016108

Hertenstein, M. J., Keltner, D., App, B., Bulleit, B. A., and Jaskolka, A. R. (2006). Touch Communicates Distinct Emotions. Emotion 6 (3), 528-533. doi:10.1037/ 1528-3542.6.3.528

Hertenstein, M. J. (2002). Touch: Its Communicative Functions in Infancy. Hum. Dev. 45 (2), 70-94. doi:10.1159/000048154

Hirche, S., and Buss, M. (2012). Human-oriented Control for Haptic Teleoperation. Proc. IEEE 100 (3), 623-647. doi:10.1109/JPROC.2011.2175150

Hoggan, E. (2013). "Haptic Interfaces," in The Sage Handbook of Digital Technology Research. Editors S. Price, C. Jewitt, and B. Brown (London: Sage), 342-358.

Hoshi, T., Takahashi, M., Iwamoto, T., and Shinoda, H. (2010). Noncontact Tactile Display Based on Radiation Pressure of Airborne Ultrasound. IEEE Trans. Haptics 3 (3), 155-165. july-september 2010. doi:10.1109/toh.2010.4

Huisman, G., and Darriba Frederiks, A. (2013). "Towards Tactile Expressions of Emotion through Mediated Touch," in CHI'13 Extended Abstracts on Human Factors in Computing Systems, 1575-1580. doi:10.1145/2468356.2468638

Huisman, G. (2017). Social Touch Technology: A Survey of Haptic Technology for Social Touch. IEEE Trans. Haptics 10 (Jul. 2017), 3391-3408. doi:10.1109/ TOH.2017.2650221

Huxley, A. (1932). Brave New World. London: Chatto \& Windus.

Ipakchian Askari, S., Harjunen, V., Haans, A., Ravaja, N., and Ijsselsteijn, W. (2020). Does Mediated Social Touch Succesfully Approximate Natural Social Touch? Annu. Rev. cybertherapy Telemed. 2020, 99.

Jakubiak, B. K., and Feeney, B. C. (2017). Affectionate Touch to Promote Relational, Psychological, and Physical Well-Being in Adulthood: A Theoretical Model and Review of the Research. Pers Soc. Psychol. Rev. 21 (3), 228-252. doi:10.1177/1088868316650307

Jewitt, C., Price, S., Mackley, K., Yiannoutsou, N., and Atkinson, D. (2020). Interdisciplinary Insights for Digital Touch Communication. London: Springer-Nature.

Jones, L. A. (2018). Haptics. MIT Press Essential Knowledge series.

Kamleitner, B., and Mitchell, V. (2019). Your Data Is My Data: A Framework for Addressing Interdependent Privacy Infringements. J. Public Pol. Marketing 38 (4), 433-450. doi:10.1177/0743915619858924

Kim, H.-K., Lee, S., and Yun, K.-S. (2011). Capacitive Tactile Sensor Array for Touch Screen Application. Sensors Actuators A: Phys. 165 (1), 2-7. doi:10.1016/ j.sna.2009.12.031

Kwon, D.-S., Yang, T.-H., and Cho, J. (2010). "Trend \& Prospects of Haptic Technology in mobile Devices," in 2010 IEEE International Symposium on Industrial Electronics (IEEE), 3778-3783. doi:10.1109/ISIE.2010.5637683

Lanier, J. (2010). You Are Not a Gadget: A Manifesto. New York: Alfred A. Knopf.

Leonardis, D., Solazzi, M., Bortone, I., and Frisoli, A. (2015). "A Wearable Fingertip Haptic Device with 3 DoF Asymmetric 3-RSR Kinematics," in IEEE World Haptics Conference (New York, USA: WHC), 388-393. doi:10.1109/WHC.2015.7177743

Light, K. C., Grewen, K. M., and Amico, J. A. (2005). More Frequent Partner Hugs and Higher Oxytocin Levels Are Linked to Lower Blood Pressure and Heart Rate in Premenopausal Women. Biol. Psychol. 69 (Apr), 15-21. doi:10.1016/ j.biopsycho.2004.11.002

Limerick, H., Hayden, R., Beattie, D., Georgiou, O., and Müller, J. (2019). "User Engagement for Mid-air Haptic Interactions with Digital Signage," in Proceedings of the 8th ACM International Symposium on Pervasive Displays (PerDis '19), New York, NY, USA (New York, USA: Association for Computing Machinery), 1-7. Article 15. doi:10.1145/3321335.3324944

Linden, D. (2015). Touch: The Science of Hand, Heart and Mind. New York, USA: Viking Books.

Löken, L. S., Wessberg, J., Morrison, I., McGlone, F., and Olausson, H. (2009). Coding of Pleasant Touch by Unmyelinated Afferents in Humans. Nat. Neurosci. 12 (5), 547-548. doi:10.1038/nn.2312

MacLean, K. E., Schneider, O. S., and Seifi, H. (2017). Multisensory Haptic Interactions: Understanding the Sense and Designing for it. Assoc. Comput. Machinery Morgan Claypool, 97-142. doi:10.1145/3015783.3015788 
Marinetti, F. T. (1921). The Manifesto of Tactilism. Milan. 11 January 1921.

McGlone, F., Wessberg, J., and Olausson, H. (2014). Discriminative and Affective Touch: Sensing and Feeling. Neuron 82 (4), 737-755. doi:10.1016/ j.neuron.2014.05.001

Meier, A., Matthies, D. J. C., Urban, B., and Wettach, R. (2015). "Exploring Vibrotactile Feedback on the Body and Foot for the Purpose of Pedestrian Navigation," in Proceedings of the 2nd International Workshop on Sensor-Based Activity Recognition and Interaction. doi:10.1145/2790044.2790051

Miller, M. R., Herrera, F., Jun, H., Landay, J. A., and Bailenson, J. N. (2020). Personal Identifiability of User Tracking Data during Observation of 360degree VR Video. Sci. Rep. 10 (1), 1-10. doi:10.1038/s41598-020-74486-y

Morrison, I. (2016). Keep Calm and Cuddle on: Social Touch as a Stress Buffer. Adaptive Hum. Behav. Physiol. 2, 344-362. doi:10.1007/s40750-016-0052-x

Nittala, A. S., Withana, A., Pourjafarian, N., and Steimle, J. (2018). Multi-Touch Skin. Proc. 2018 CHI Conf. Hum. Factors Comput. Syst. - CHI '18, 1-12. doi:10.1145/3173574.3173607

Obrist, H. (2010). Manifestos for the Future. Eflux Journal \#12. Retrieved January 82017 from.

Obrist, M., Subramanian, S., Gatti, E., Long, B., and Carter, T. (2015). "Emotions Mediated through Mid-air Haptics," in Proceedings of the 33rd Annual ACM Conference on Human Factors in Computing Systems, New York, NY, USA (New York, USA: Association for Computing Machinery), 2053-2062. doi:10.1145/ 2702123.2702361

Olausson, H., Wessberg, J., Morrison, I., McGlone, F., and Vallbo, Å. (2010). The Neurophysiology of Unmyelinated Tactile Afferents. Neurosci. Biobehavioral Rev. 34 (2), 185-191. doi:10.1016/j.neubiorev.2008.09.011

Ornati, M. (2022). Size Me Up. The datafication of dress embodiment in fashion ecommerce fit applications. International Journal of Fashion Studies. Bristol, UK: Intellect.

Orth, M., Post, R., and Cooper, E. (1998). "Fabric Computing Interfaces," in CHI 98 Conference Summary on Human Factors in Computing Systems, 331-332. doi:10.1145/286498.286800

Owen, P. M., and Gillentine, J. (2011). Please Touch the Children: Appropriate Touch in the Primary Classroom. Early Child. Dev. Care 181 (6), 857-868. doi:10.1080/03004430.2010.497207

Ozolins, A., and Sandberg, C. (2009). "Development of a Multifactor Scale Measuring the Psychological Dimensions of Touch Avoidance," in International Journal of Psychology: A Biopsychosocial Approach, 3, 33-56.

Pacchierotti, C., Sinclair, S., Solazzi, M., Frisoli, A., Hayward, V., and Prattichizzo, D. (2017). Wearable Haptic Systems for the Fingertip and the Hand: Taxonomy, Review, and Perspectives. IEEE Trans. Haptics 10 (4), 580-600. doi:10.1109/toh.2017.2689006

Parisi, D. (2018). Archaeologies of Touch: Interfacing with Haptics from Electricity to Computing. Minnesota, USA: University of Minnesota Press.

Parisi, D., and Farman, J. (2019). Tactile Temporalities: the Impossible Promise of Increasing Efficiency and Eliminating Delay through Haptic media. Convergence 25 (1), 40-59. doi:10.1177/1354856518814681

Parisi, D., Paterson, M., and Archer, J. E. (2017)., 19. London, UK: Sage, 1513-1522. doi:10.1177/1461444817717518Haptic media StudiesNew Media Soc. 10

Pihkala, S., Huuki, T., and Sunnari, V. (2019). Moving with Touch: Entanglements of a Child, Valentine's Day Cards, and Research-Activism against Sexual Harassment in Pre-teen Peer Cultures. Soc. Sci. 8, 226. doi:10.3390/ socsci 8080226

Pourjafarian, N., Withana, A., Paradiso, J. A., and Steimle, J. (2019). "Multi-Touch Kit," in UIST '19: Proc. of the 32nd Annual ACM Symposium on User Interface Software and Technology, 1071-1083. doi:10.1145/3332165.3347895

Prasad, M., Taele, P., Goldberg, D., and Hammond, T. A. (2014). "Haptimoto: Turn-By-Turn Haptic Route Guidance Interface for Motorcyclists," in Proceedings of the SIGCHI Conference on Human Factors in Computing Systems (New York, USA: ACM), 3597-3606.

Prattichizzo, D. (2021). Beyond the Pandemic: The Role of Haptics in Defining the New Normal. IEEE Trans. Haptics 14 (1), 1. doi:10.1109/ TOH.2021.30657721

Prattichizzo, D., Pacchierotti, C., and Rosati, G. (2012). Cutaneous Force Feedback as a Sensory Subtraction Technique in Haptics. IEEE Trans. Haptics 5, 289-300. doi:10.1109/TOH.2012.15
Price, S., Jewitt, C., and Yiannoutsou, N. (2021). Conceptualising Touch in VR. Virtual Reality 25, 863-877. doi:10.1007/s10055-020-00494-y

Rakkolainen, I., Freeman, E., Sand, A., Raisamo, R., and Brewster, S. (2021). A Survey of Mid-air Ultrasound Haptics and its Applications. IEEE Trans. Haptics 14 (1), 2-19. doi:10.1109/TOH.2020.30187541

Rantala, J., Salminen, K., Raisamo, R., and Surakka, V. (2013). Touch Gestures in Communicating Emotional Intention via Vibrotactile Stimulation. Int. J. Human-Computer Stud. 71 (6), 679-690. doi:10.1016/ j.ijhcs.2013.02.004

Remland, M. S., Jones, T. S., and Brinkman, H. (1991). Proxemic and Haptic Behavior in Three European Countries. J. Nonverbal Behav. 15 (4), 215-232. doi:10.1007/bf00986923

Republic Francaise (1994). LAW N ${ }^{\circ} 94-665$ of August 4, 1994 Relating to the Use of the French Language. Available at: https://www.legifrance.gouv.fr/jorf/id/ JORFTEXT000000349929

Roberts, P., Damian, D. D., Shan, W., Lu, T., and Majidi, C. (2013). "Soft-matter Capacitive Sensor for Measuring Shear and Pressure Deformation," in 2013 IEEE International Conference on Robotics and Automation, 3529-3534. doi:10.1109/ICRA.2013.6631071

Romanus, T., Frish, S., Maksymenko, M., Frier, W., Corenthy, L., and Georgiou, O. (2019). "Mid-Air Haptic Bio-Holograms in Mixed Reality," in 2019 IEEE International Symposium on Mixed and Augmented Reality Adjunct (New York, USA: ISMAR-Adjunct)), 348-352. doi:10.1109/ISMARAdjunct.2019.00-14

Sale, J. E. M., and Thielke, S. (2018). Qualitative Research Is a Fundamental Scientific Process. J. Clin. Epidemiol. 102, 129-133. doi:10.1016/ j.jclinepi.2018.04.024

Saleme, E. B., Covaci, A., Mesfin, G., Santos, C. A. S., and Ghinea, G. (2019). Mulsemedia DIY: A Survey of Devices and a Tutorial for Building Your Own Mulsemedia Environment. ACM Comput. Surv.Surveys 52 (3), 1-29. Article 58. doi: $10.1145 / 3319853$

Schneider, O., MacLean, K., Swindells, C., and Booth, K. (2017). Haptic Experience Design: What Hapticians Do and where They Need Help. Int. J. HumanComputer Stud. 107, 5-21. doi:10.1016/j.ijhcs.2017.04.004

Seifi, H., Fazlollahi, F., Oppermann, M., Sastrillo, J. A., Ip, J., Agrawal, A., Park, G., Kuchenbecker, K. J., and MacLean, K. E. (2019). "Haptipedia,". Paper 558 in Proceedings of the 2019 CHI Conference on Human Factors in Computing Systems, 1-12. doi:10.1145/3290605.3300788

Shah, N., Basteris, A., and Amirabdollahian, F. (2014). Design Parameters in Multimodal Games for Rehabilitation. Games Health J. 3 (1), 13-20. doi:10.1089/g4h.2013.0044

Sorokowska, A., Saluja, S., Sorokowski, P., Frąckowiak, T., Karwowski, M., Aavik, T., et al. (2021). Affective Interpersonal Touch in Close Relationships: A CrossCultural Perspective. Personal. Soc. Psychol. Bull., 0146167220988373.

Srinivasan, M. A., and Basdogan, C. (1997). Haptics in Virtual Environments: Taxonomy, Research Status, and Challenges. Comput. Graphics 21 (4), 393-404. doi:10.1016/S0097-8493(97)00030-7

Srivastava, P., and Hopwood, N. (2009). A Practical Iterative Framework for Qualitative Data Analysis. Int. J. Qual. Methods 8, 76-84. doi:10.1177/ 160940690900800107

Stone, R. J. (2001). "Haptic Feedback: A Brief History from Telepresence to Virtual Reality," in International Workshop on Haptic HumanComputer Interaction, Berlin, Heidelberg (Springer), 1-16. doi:10.1007/3-540-44589-7_1

Strohmeier, P., Güngör, S., Herres, L., Gudea, D., Fruchard, B., and Steimle, J. (2020). "Barefoot: Generating Virtual Materials Using Motion Coupled Vibration in Shoes," in Proceedings of the 33rd annual acm symposium on user interface software and technology. New York, USA. doi:10.1145/3379337.3415828

Strohmeier, P., and Hornbæk, K. (2017). "Generating Haptic Textures with a Vibrotactile Actuator," in Proceedings of the 2017 CHI Conference on Human Factors in Computing Systems, New York, NY, USA (Association for Computing Machinery), 4994-5005. doi:10.1145/3025453.3025812

Sutherland, I. E. (1965). "The Ultimate Display," in Proceedings of the IFIP Congress, 506-508.

Teyssier, M., Bailly, G., Pelachaud, C., and Lecolinet, E. (2020). Conveying Emotions through Device-Initiated Touch. IEEE Trans. Affective Comput., 1. doi:10.1109/TAFFC.2020.3008693 
Tibbits, S. (2017). Active Matter. MIT Press.

Van Erp, J. B. F., Kyung, K.-U., Kassner, S., Carter, J., Brewster, S., Weber, G., and Andrew, I. (2010). "Setting the Standards for Haptic and Tactile Interactions: ISO's Work," in International Conference on Human Haptic Sensing and Touch Enabled Computer Applications, Berlin, Heidelberg (Springer), 353-358. doi:10.1007/978-3-642-14075-4_52

van Erp, J. B. F., and Toet, A. (2015). Social Touch in Humanâ€"Computer Interaction. Front. Digit. Humanit. 2, 1-14. doi:10.3389/fdigh.2015.00002

Walker, S. C., Trotter, P. D., Swaney, W. T., Marshall, A., and McGlone, F. P. (2017). C-tactile Afferents: Cutaneous Mediators of Oxytocin Release during Affiliative Tactile Interactions? Neuropeptides 64, 27-38. doi:10.1016/..npep.2017.01.001

Weigel, M., Lu, T., Bailly, G., Oulasvirta, A., Majidi, C., and Steimle, J. (2015). "iSkin," in Proceedings of the 33rd Annual ACM Conference on Human Factors in Computing Systems - CHI '15, 2991-3000. doi:10.1145/2702123.2702391

Weigel, M., Mehta, V., and Steimle, J. (2014). "More Than Touch," in Proceedings of the SIGCHI Conference on Human Factors in Computing Systems, 179-188. doi:10.1145/2556288.2557239

Wessely, M., Tsandilas, T., and Mackay, W. E. (2016). "Stretchis," in Proceedings of the 29th Annual Symposium on User Interface Software and Technology UIST, 16. 697-704. doi:10.1145/2984511.2984521

Willemse, C. J. A. M., Toet, A., and van Erp, J. B. F. (2017). Affective and Behavioral Responses to Robot-Initiated Social Touch: Toward Understanding the Opportunities and Limitations of Physical Contact in Human-Robot Interaction. Front. ICT 4, 12. doi:10.3389/fict.2017.00012

Willemse, C. J. A. M., and Van Erp, J. B. F. (2019). Social Touch in Human-Robot Interaction: Robot-Initiated Touches Can Induce Positive Responses without Extensive Prior Bonding. Int. J. Soc. Robotics 11 (2), 285-304. doi:10.1007/s12369-018-0500-9
Withana, A., Groeger, D., and Steimle, J. (2018). "Tacttoo," in Proceedings of the 31st annual ACM symposium on user interface software and technology. New York, USA: UIST. doi:10.1145/3242587.3242645

Yu, X., Xie, Z., Yu, Y., Lee, J., Vazquez-Guardado, A., Luan, H., et al. (2019). Skinintegrated Wireless Haptic Interfaces for Virtual and Augmented Reality. Nature 575, 473-479. doi:10.1038/s41586-019-1687-0

Conflict of Interest: WF is employed by UltraLeap.

The remaining authors declare that the research was conducted in the absence of any commercial or financial relationships that could be construed as a potential conflict of interest.

Publisher's Note: All claims expressed in this article are solely those of the authors and do not necessarily represent those of their affiliated organizations, or those of the publisher, the editors and the reviewers. Any product that may be evaluated in this article, or claim that may be made by its manufacturer, is not guaranteed or endorsed by the publisher.

Copyright $\odot 2021$ Jewitt, Price, Steimle, Huisman, Golmohammadi, Pourjafarian, Frier, Howard, Ipakchian Askari, Ornati, Panëels and Weda. This is an open-access article distributed under the terms of the Creative Commons Attribution License (CC $B Y)$. The use, distribution or reproduction in other forums is permitted, provided the original author(s) and the copyright owner(s) are credited and that the original publication in this journal is cited, in accordance with accepted academic practice. No use, distribution or reproduction is permitted which does not comply with these terms. 